\title{
Update on the Molecular Epidemiology and Diagnostic Tools for Blastocystis sp
}

Tamalee Roberts $^{1,2^{*}}$, Damien Stark ${ }^{1}$, John Harkness ${ }^{1}$ and John Ellis ${ }^{2}$

${ }^{1}$ Department of Microbiology, SydPath, St. Vincent's Hospital, Victoria st, Darlinghurst, N.S.W, Australia

${ }^{2}$ School of Medical and Molecular Sciences, University of Technology, Sydney, Ultimo, N.S.W, Australia

*Corresponding author: Tamalee Roberts, Department of Microbiology, St. Vincent's Hospital, Darlinghurst, N.S.W, Australia, Tel: 61283829209; Fax: 61283822989; E-mail: troberts@stvincents.com.au

Rec Date: Jan 14, 2014, Acc date: Apr 24, 2014, Pub date: Apr 26, 2014

Copyright: (c) 2014 Robert T, et al. This is an open-access article distributed under the terms of the Creative Commons Attribution License, which permits unrestricted use, distribution, and reproduction in any medium, provided the original author and source are credited.

\begin{abstract}
Blastocystis is the most common enteric protist found in humans. Due to the recent advancements in molecular technologies, up to 17 subtypes have been identified from humans and animals. Molecular epidemiological studies have shown the large range of subtype (ST) distribution within geographical locations with ST1-9 being identified in humans. ST3 has been identified as the predominant subtype in most epidemiological studies with a considerable absence of ST4 noted in Africa and the Middle East compared to Europe and Australia where this subtype is fairly common. This review summarises the molecular tools used for diagnosis and speciation of Blastocystis and comments on advancements in the area over the last 15 years and what future trends may be. This review also describes the geographical distribution of Blastocystis and comments on possible intra-subtype evolutionary relationships.
\end{abstract}

Keywords Blastocystis sp.; Epidemiology; Subtyping; Diagnosis

\section{Introduction}

For a protist that was discovered over 100 years ago there is still so much unknown about Blastocystis in regards to its pathogenicity, transmission, lifecycle and susceptibility to antimicrobials. Symptoms associated with Blastocystis infection include diarrhoea, abdominal pains and bloating $[1,2]$. Until recently it was not possible to differentiate between the subtypes of Blastocystis and many different molecular techniques have been developed to distinguish between them. In turn this has resulted in different terminologies being used for the identification and designation of Blastocystis subtypes. So far 17 subtypes have been identified within the Blastocystis clades existing in mammals, birds and reptiles. Due to recent studies it has been shown that no group exclusive to humans exists and that human isolates predominantly lie within subtype 1-4 [3-5]. Until recently, all human isolates of Blastocystis were commonly referred to as Blastocystis hominis and all other animal isolates were called Blastocystis sp. but these recent molecular results now show that all samples identified through only microscopy should be called Blastocystis sp. due to there not being a single subtype specific to humans. From many early studies completed on the subtyping of Blastocystis, lack of consistency in the designation of subtypes resulted in confusion and made it difficult for comparison and collaboration within studies. A study on the consensus for the terminology of Blastocystis compared all the previously published data which showed many discrepancies with subtype allocation [6]. This paper proposed that all isolates fall within nine subtypes and concluded that a standardisation within the nomenclature would make future epidemiological and clinical studies much easier. Since this paper was published, a further eight subtypes have been identified from animals [7-9]. Recently the whole genome sequence for subtype (ST) 7 was completed. This new information has shed some light on many of the aspects of Blastocystis including the metabolic pathways, virulence factors and possible drug resistance mechanisms.

\section{Typing Methods}

Clark and colleagues were among the first to employ molecular techniques to distinguish the various subtypes of Blastocystis present in human stool samples. They used Restriction Fragment Length Polymorphism (RFLP) analysis of Polymerase Chain Reaction (PCR) amplified small subunit (SSU) rDNA for the rapid comparison of multiple isolates. Through this technique they identified seven different Blastocystis ribodemes, and suggested that Blastocystis was a zoonosis [10]. In the last 15 years there has been extensive work done in developing molecular techniques to distinguish the different subtypes of Blastocystis and 17 subtypes have been identified from humans and animals. Using PCR and RFLP analysis, which relies on the digestion of PCR products with restriction endonucleases to yield variable gel banding products, up to five different subtypes were identified from different geographical locations [11-14]. The use of RFLP can be problematic in that mixed infections may be missed and if there is a mutation at any of the bases that make up the restriction recognition sequence isolates could be incorrectly identified [15].

The application of subtype- specific primer pairs was developed to distinguish between seven different subtypes but as more subtypes have been identified this method for typing is not reliable as it does not cover all the subtypes and therefore infections may be missed or incorrectly assigned to a different subtype [16-18]. PCR with subsequent dideoxy sequencing of amplified PCR products, targeting typically the $18 \mathrm{~S}$ SSU rRNA, is now the most common way for identifying the different subtypes isolated from samples as this method allows for all subtypes to be identified, given that correct primer pairs have been chosen that do not show subtype preference [4].

Pyrosequencing technology combined with a nested PCR was developed to differentiate between seven subtypes [15]. This system is based on the pyrophosphate released when a nucleotide is introduced to the DNA-strand and is suited for detecting single or multiple nucleotide polymorphisms within short-read DNA sequences $[19,20]$. This technique shows a high specificity and sensitivity and is a fairly 
fast typing method. This technique does have problems though with possible nucleotide miscalling in short reads and is also not possible to discriminate between subtypes found in mixed infections.

There have only been three studies reported which have developed a real-time (RT) PCR for Blastocystis. The first study [21] was able to detect ST1, ST3 and ST4 but it is unknown whether other subtypes are able to be detected using this assay and therefore the specificity and sensitivity is unknown. The second study designed a genus- specific PCR which was able to detect all known subtypes so far identified in humans [22]. This test only had $95 \%$ specificity though, and the amplicon size of $339 \mathrm{bp}$ is much longer than usually desired for RTPCR sensitivity. The most recent study developed a TaqMan assay which is highly sensitive and specific for subtypes 1-9 of Blastocystis [23]. This appears to be the most sensitive and specific RT-PCR so far developed but still does not differentiate between the subtypes. At the moment it appears that RT-PCR could be the most useful tool for the screening of Blastocystis but subtyping of isolates by conventional PCR is still the only way to designate subtypes. The development of RT- PCR techniques which have the ability to discriminate between the different subtypes within a sample will be beneficial for screening and epidemiological studies for Blastocystis. RT-PCR would also prove to be more time and cost effective with extraction and RT-PCR theoretically being able to be completed within only a few hours.

More recently Multilocus Sequence Typing (MLST) of the Mitochondria-Like Organelle (MLO) of Blastocystis has been used to show the differences in diversity within subtypes [24]. There were five loci of ST3 MLST sequences studied and six loci for the ST4 MLST. The authors showed that most human ST3 infections occur through human- human transmission as most human infections were restricted to a single clade (MLO clade 1) within the ST3 group. Animal infections and people exposed to animals (in particular animal handlers) were found to lie within three other clades which show the result of zoonotic transmission. This study showed the intra-subtype genetic variability within ST3 and ST4. The study also suggested that ST4 has a more recent history of colonising humans than ST3 due to the lack of variation within the sequences and also because ST4 is common in European populations but is rarely found elsewhere.

The use of the "barcode region" for sequencing appears to be the most effective for the designation of Blastocystis subtypes and is the best technique used if phylogenetic information is to be obtained [4]. DNA barcoding refers to a method proposed to produce a unique identifier for all living species and has been used previously to study a variety of animals $[25,26]$. The Blastocystis barcode region amplifies the 5' $600 \mathrm{bp}$ region of the Blastocystis SSU-rDNA using the primer pair of RD5, a primer with broad eukaryotic specificity, and BhRDr which is more specific to Blastocystis and was designed for subsequent sequencing. This PCR does have the possibility for false positives though if only used for Blastocystis screening. The PCR has the ability to detect other eukaryotes particularly fungi which makes this technique not desirable when sequencing of positives is not available.

A substantial number of sequences in Genbank use the "barcode region" and have been submitted to the Blastocystis Subtype (18S) and Sequence Typing (MLST) Database (www.pubmlst.org/blastocystis) which makes comparison and designation of subtypes simpler and keeps integrity within the subtype allocations. The advantage of the Blastocystis Subtype (18S) and Sequence Typing (MLST) Database is that it uses the consensus subtype nomenclature (discussed below), unlike GenBank, and also assigns the allele to the SSU-rDNA barcode sequence [27]. This database currently has 255 sequences and 64 MLST profiles and is continually updated.

The uses of matrix-assisted laser desorption/ionisation time-offlight mass spectrometry (MALDI-TOF MS) has recently revolutionised the clinical microbiology laboratory with accurate, rapid results available for the identification of microorganisms. A recent study showed the accuracy of MALDI-TOF MS for the speciation of five different Blastocystis STs [28]. Axenic cultures speciated by SSU-rDNA analysis were used to create profiles for the different Blastocystis subtypes. It was found that speciation was better after extraction using the ethanol/formic acid procedure. Axenic cultures were shown to be the preferred sample used with xenic broth cultures not having as high ID scores. This study did have a small study number though and showed the limitations of the xenic cultures. This does however highlight an exciting area of research for the future which could substantially cut costs for Blastocystis detection and speciation.

Before 2007 there was not a standardised subtype nomenclature and several studies assigned the same subtypes to different groups creating some confusion and meaning an overall global picture of Blastocystis molecular epidemiology was not possible. A consensus was agreed upon in 2007 and since then a greater picture of subtype infection has been possible [6]. Although there is now a standardised nomenclature, there is still not a standardised method for subtyping with people doing sequencing on different regions of the SSU rDNA or using Sequence-Tagged-Site PCR for subtype designation. Through the application of these different techniques it has been shown that there is substantial antigenic and genetic heterogeneity among Blastocystis isolates within and among geographical regions. Special care must be taken when choosing the primers used in PCR where certain primers can have an effect on the final result. Some primers have specific subtype amplification preferential, which could result in other subtypes being missed in the analysis. Also some primers have been developed from cultured samples, whereas others work better with direct extraction of DNA from the stool so this needs to be taken in to consideration when using molecular techniques for diagnosis. The primers that are being used may have different sensitivity depending on what sort of sample is being used and may give an incorrect result. There is still much more work needed to develop a simple and effective method for the detection and typing of Blastocystis sp. A RTPCR that is able to detect and allocate all of the subtypes would be the most useful for diagnosis as well as epidemiological studies whereas the "barcode region" may be the most effective technique for phylogenetic studies and showing evolutionary relationships.

\section{Epidemiology}

The prevalence of Blastocystis infection was stated as being higher in developing countries than in developed countries [29]. Due to varying modes of diagnostic techniques used in epidemiological studies of Blastocystis, it is difficult to determine the actual prevalence of Blastocystis due to some studies relying solely on microscopy for diagnosis. It was shown that molecular techniques have the highest sensitivity rates for diagnosis of this organism [30,31] and the use of only microscopy in diagnosis and epidemiological studies limits interpretation of data on prevalence of Blastocystis infections.

Through the use of molecular techniques for epidemiological studies, ST3 was found to be the predominant subtype isolated in humans with a range of $40-92 \%$ of isolates belonging to this group 
$[10,13,32]$. There have been two studies where ST1 was the predominant subtype with $51.4 \%$ of positive from this group belonging to this group in China, $41 \%$ in Brazil $[17,33,34]$ and three others where ST4 was the predominant subtype with $63 \%$ in France, $84 \%$ in Nepal and $94.1 \%$ in Spain, with no ST3 identified in the Spain population $[22,35,36]$. From South American countries it is seen that there is a predominance of ST1, ST2 and ST3 with no other STs being isolated. ST1 appears to be the predominant ST from these South American studies followed by ST2 then ST3 with no ST4 noted [34,37].

In Singapore the prevalence of Blastocystis is at 3.3\% of the studied population with ST1 and ST3 being the only subtypes isolated. There was a $78 \%$ incidence of ST3 and a $22 \%$ incidence of ST1. This prevalence of ST3 in humans in an urbanised area such as Singapore is consistent with the argument that ST3 is of human origin [38].

An investigation of isolates of Blastocystis in Japan, Pakistan, Bangladesh, Germany and Thailand showed that ST3 was the most common varying from 41.7-92.3\% positives with the second most common being ST1 (7.7-25\%) and ST4 (10-22.9\%). In Bangladesh an almost equal number of symptomatic and asymptomatic isolates were tested but there was no major percentage difference between ST1 and ST3 so it is not possible to comment on the pathogenicity of these two subtypes [18].

The incidence of Blastocystis in a province in China showed 32.6\% of people studied were positive for Blastocystis. ST3 was the predominant subtype in $70.5 \%$ of the positive population, followed by ST1 with $20.5 \%$. The significance of hygienic practices was shown to have an effect on the type of infection with ST3 corresponding to the drinking of unboiled water and ST1 relating to the consumption of raw water plants [39].

In Lebanon there was a $19 \%$ incidence of Blastocystis from a population of symptomatic and asymptomatic people [40]. Four subtypes were identified with almost equal numbers of ST3 (33.3\%), ST2 (33.3\%) and ST1 (30.6\%) and only one patient with ST4 (2.8\%). There was an association seen between ST1 and symptoms.

A study performed to show the incidence of intestinal parasites among immigrants in food handling and housemaid jobs in Qatar showed that of the 1337 people surveyed from the Philippines, Indonesia, Indian sub-continent and Africa over two years, there were 588 individuals positive for at least one of the parasites studied. Of all the parasites studied, Blastocystis was shown to have the second highest incidence rate at $13.2 \%$ for all the nationalities put together with only a slightly higher incidence in African samples than the other nationalities. There was a much higher incidence in women than men [41].

A study of an aborigine community in Pahang, Malaysia showed that Blastocystis had the highest incidence rate $(52.3 \%)$ followed by G. lamblia (29.2\%). Overall there was a $72.3 \%$ positive parasitic infection rate for this community. These high rates of parasitic infection can be attributed to the low standard of sanitation and hygiene in this area with there being no electricity, running water or toilets inside their dwellings and water from the river being used for all purposes including cooking, bathing, swimming and washing vegetables [42].

In a Zambian school, $53.8 \%$ of children were reported to be infected with Blastocystis with some being co-infected with Endolimax nana (which is another parasite not considered to be pathogenic to humans) with almost half the children suffering from diarrhoea. This study supports the argument that Blastocystis infections are associated with inadequate sanitation and low hygienic standards and can contribute to diarrhoea in children in developing countries [43].

For travellers, it was shown that there is an increased rate of infection while travelling to tropical and under-developed countries, with $30 \%$ infection rates found in tourists in Nepal. It is frequently identified in stools from foreign workers in South-east Asia and Taiwan, with the incidence of infection being attributed to poor personal and environmental hygiene, lack of water supply and poor sewage and rubbish removal [44]. For 36 Peace Corps volunteers (PCV's) in Guatemala for a length of two years or greater there was a $38.8 \%$ infection rate with an intestinal parasite. Blastocystis was the most common parasite in these samples at $77.8 \%$ with a progressive increase in infection from $11.1 \%$ of PCV's at the start to $37.9 \%$ and then $53.1 \%$ and then $77.8 \%$ at the end of the 2 year study. Most of the Blastocystis infections were found to be asymptomatic and last several weeks. The prevalence of intestinal parasites found in these PCV's in Guatemala could be attributed to them being repeatedly exposed to potentially risky diets and diarrheal illness is the most common medical disorder encountered by travellers from developed countries to developing countries [45].

In an orphanage in Thailand over a one year period the rate of infection varied between $6.6 \%-27.4 \%$ with samples being taken every two months [46]. These varying results could have been due to the shedding effect seen in many cases of parasitic infection as well as the diagnostic technique (microscopy of a permanent smear and culture) being used not being sensitive enough. These results also highlight the easy transmission of Blastocystis in places with poor hygiene. Table 1 summarises the different subtypes isolated in different countries.

\begin{tabular}{|c|c|c|c|c|c|c|c|c|c|c|c|c|}
\hline \multirow{2}{*}{$\begin{array}{l}\text { Country/region (no. Of } \\
\text { individuals studied) }\end{array}$} & \multirow{2}{*}{$\begin{array}{ll}\text { No. } & \text { Of } \\
\text { positive } & \\
\text { isolates } & \end{array}$} & \multicolumn{10}{|c|}{ Subtypes identified (\%) } & \multirow[t]{2}{*}{ References } \\
\hline & & 1 & 2 & 3 & 4 & 5 & 6 & 7 & 8 & 9 & unknown/ mixed & \\
\hline Australia (513) & 91 & 28 & 5 & 40 & 12 & 0 & 6 & 1 & 2 & 0 & 0 & [47] \\
\hline Australia & 12 & 41 & 0 & 33 & 16 & 0 & 8 & 0 & 0 & 0 & 8 & [48] \\
\hline Bangladesh & 26 & 7.7 & 0 & 92.3 & 0 & 0 & 0 & 0 & 0 & 0 & 0 & [18] \\
\hline Brazil (382) & 66 & 41 & 32 & 17 & 0 & 0 & 0 & 0 & 0 & 0 & 10 & [34] \\
\hline China, Yunnan (239) & 78 & 20.5 & 1.3 & 70.5 & 1.3 & 0 & 0 & 0 & 0 & 0 & 5 & [39] \\
\hline Colombia & 125 & 56 & 32 & 12 & 0 & 0 & 0 & 0 & 0 & 0 & 0 & [37] \\
\hline
\end{tabular}


Citation: $\quad$ Roberts T, Stark D,Harkness J, Ellis J (2014) Update on the Molecular Epidemiology and Diagnostic Tools for Blastocystis sp. J Med

Page 4 of 6

\begin{tabular}{|c|c|c|c|c|c|c|c|c|c|c|c|c|}
\hline Denmark & 29 & 3.4 & 20.7 & 51.7 & 24.1 & 0 & 0 & 0 & 0 & 0 & 0 & [49] \\
\hline Denmark (126) & 24 & 8.3 & 12.5 & 25 & 37.5 & 0 & 0 & 0 & 0 & 12.5 & 0 & {$[50]$} \\
\hline Denmark (444) & 25 & 4 & 16 & 4 & 76 & 0 & 0 & 0 & 0 & 0 & 0 & [51] \\
\hline Egypt & 44 & 18.2 & 0 & 54.5 & 0 & 0 & 18 & 9.1 & 0 & 0 & 0 & [52] \\
\hline Egypt & 100 & 15 & 0 & 49 & 0 & 0 & 33 & 13 & 0 & 0 & 10 & [53] \\
\hline France & 40 & 25.6 & 9.3 & 53.5 & 9.3 & 0 & 0 & 2.3 & 0 & 0 & 7.5 & [32] \\
\hline Germany (67) & 12 & 25 & 0 & 41.7 & 0 & 17 & 0 & 17 & 0 & 0 & 0 & [18] \\
\hline Germany & 171 & 23.4 & 0.6 & 69 & 7 & 0 & 0 & 0 & 0 & 0 & 3 & [12] \\
\hline Greece & 45 & 20 & 13.3 & 60 & 2.2 & 0 & 2.2 & 2.2 & 0 & 0 & 0 & [54] \\
\hline Italy (34) & 34 & 8.8 & 20.6 & 47.1 & 17.7 & 0 & 0 & 2.9 & 2.9 & 0 & 4 & [55] \\
\hline Iran & 40 & 50 & 10 & 40 & 0 & 0 & 0 & 0 & 0 & 0 & 12 & [56] \\
\hline Iran & 141 & 34 & 23 & 37 & 0 & 0 & 0 & 5 & 0 & 0 & 23 & [57] \\
\hline Japan & 64 & 15.6 & 20.3 & 48.4 & 10.9 & 0 & 0 & 0 & 0 & 0 & 4.7 & [13] \\
\hline Japan $(2,037)$ & 50 & 8 & 0 & 52 & 4 & 0 & 22 & 10 & 0 & 4 & 0 & [18] \\
\hline Lebanon (220) & 42 & 30.6 & 33.3 & 33.3 & 2.8 & 0 & 0 & 0 & 0 & 0 & 0 & [40] \\
\hline Liberia & 25 & 28 & 28 & 32 & 12 & 0 & 0 & 0 & 0 & 0 & 20 & [9] \\
\hline Libya & 38 & 50 & 7.9 & 39.5 & 0 & 0 & 0 & 2.6 & 0 & 0 & 0 & [9] \\
\hline Malaysia & 40 & 12.5 & 5 & 50 & 27.5 & 0 & 0 & 0 & 0 & 0 & 2 & [58] \\
\hline Nepal (82) & 20 & 20 & 20 & 60 & 0 & 0 & 0 & 0 & 0 & 0 & 0 & [59] \\
\hline Nepal (241) & 63 & 63.5 & 12.7 & 0 & 84.1 & 0 & 0 & 0 & 0 & 0 & 70 & [36] \\
\hline Netherlands (442) & 103 & 22 & 22 & 42 & 0 & 0 & 1 & 1 & 0 & 0 & 0 & [60] \\
\hline Nigeria & 22 & 45.5 & 0 & 40.9 & 13.6 & 0 & 0 & 0 & 0 & 0 & 0.4 & [9] \\
\hline Pakistan & 10 & 20 & 0 & 70 & 0 & 0 & 10 & 0 & 0 & 0 & 0 & [18] \\
\hline Philippines & 5 & 20 & 0 & 80 & 0 & 0 & 0 & 0 & 0 & 0 & 0 & [61] \\
\hline Singapore (276) & 9 & 22.2 & 0 & 77.8 & 0 & 0 & 0 & 0 & 0 & 0 & 0 & [38] \\
\hline Spain & 51 & 2 & 3.9 & 0 & 94.1 & 0 & 0 & 0 & 0 & 0 & 0 & [35] \\
\hline Sweden & 63 & 15.9 & 14.3 & 47.6 & 20.6 & 0 & 0 & 1.6 & 0 & 0 & 0 & [62] \\
\hline Thailand (30) & 5 & 40 & 0 & 40 & 0 & 0 & 20 & 0 & 0 & 0 & 20 & [18] \\
\hline Thailand (675) & 127 & 77.9 & 22.1 & 0 & 0 & 0 & 0 & 0 & 0 & 0 & 0 & [63] \\
\hline Turkey (286) & 92 & 20 & 24 & 59.7 & 0 & 0 & 0 & 0 & 0 & 0 & 4 & [64] \\
\hline Turkey & 87 & 9.2 & 13.8 & 75.9 & 1.1 & 0 & 0 & 0 & 0 & 0 & 0 & [65] \\
\hline Turkey & 25 & 36 & 24 & 10 & 0 & 0 & 0 & 0 & 0 & 0 & 0 & [66] \\
\hline UK & 55 & 5.4 & 16.4 & 40 & 31 & 0 & 0 & 1.8 & 5.4 & 0 & 0 & [4] \\
\hline UK & 271 & 12.5 & 9.6 & 42.1 & 31.4 & 0.7 & 0.4 & 1.5 & 1.8 & 0 & 0 & [9] \\
\hline USA & 21 & 11.1 & 0 & 66.7 & 0 & 0 & 0 & 0 & 0 & 0 & 22.2 & [2] \\
\hline
\end{tabular}

Table 1: Subtype classification of human Blastocystis sp. isolates from different countries 
From all the epidemiological studies a pattern can be seen where there is a much higher incidence of ST4 in Europe and Australia compared to Africa, the Middle East and South America where very low numbers, if any, are seen. Why this is occurring is unknown, but it could be due to several reasons. The use of diagnostic tool may have an effect on the STs identified, as previously discussed, with some primer pairs showing ST preferential. Another reason may be due to the recent evolution of ST4. There is a much higher homogeny among ST4 samples compared to ST3, which is the closest related ST from phylogenetic studies, suggesting that this may be a relatively new ST [24].

\section{Conclusions}

The future of Blastocystis diagnostics and epidemiology is an exciting area. With the possibility of new genomes being available for the different subtypes, this may help improve targets for RT-PCR that will be beneficial for both diagnosis and differentiation between subtypes. As diagnosis and speciation becomes easier, more epidemiological studies will be possible which may potentially show the large range of Blastocystis distribution around the world. As more samples are studied, the relationship between geographical location and subtype might become apparent as well as intra-subtype evolutionary relationships.

\section{References}

1. Roberts T, Ellis J, Harkness J, Marriott D, Stark D (2014) Treatmen failure in patients with chronic Blastocystis infection. J Med Microbiol 63: 252-257.

2. Jones MS, Whipps CM, Ganac RD, Hudson NR, Boorom K (2009) Association of Blastocystis subtype 3 and 1 with patients from an Oregon community presenting with chronic gastrointestinal illness. Parasitol Res 104: 341-345.

3. Noel C, Dufernez F, Gerbod D, Edgcomb VP, Delgado-Viscogliosi P et al. (2005) Molecular phylogenies of Blastocystis isolates from different hosts: implications for genetic diversity, identification of species, and zoonosis. J Clin Microbiol 43: 348-55.

4. Scicluna SM, Tawari B, Clark CG (2006) DNA barcoding of blastocystis Protist 157: 77-85.

5. Parkar U, Traub RJ, Kumar S, Mungthin M, Vitali S, et al. (2007) Direct characterization of Blastocystis from faeces by PCR and evidence of zoonotic potential. Parasitology 134: 359-367.

6. Stensvold CR, Suresh GK, Tan KS, Thompson RC, Traub RJ, et al. (2007) Terminology for Blastocystis subtypes--a consensus. Trends Parasitol 23: 93-96.

7. Stensvold CR, Alfellani MA, Nørskov-Lauritsen S, Prip K, Victory EL, et al. (2009) Subtype distribution of Blastocystis isolates from synanthropic and zoo animals and identification of a new subtype. Int J Parasitol 39: 473-479.

8. Parkar U, Traub RJ, Vitali S, Elliot A, Levecke B, et al. (2010) Molecular characterization of Blastocystis isolates from zoo animals and their animal-keepers. Vet Parasitol 169: 8-17.

9. Alfellani MA, Stensvold CR, Vidal-Lapiedra A, Onuoha ES, FagbenroBeyioku AF, et al. (2013) Variable geographic distribution of Blastocystis subtypes and its potential implications. Acta Trop 126: 11-18.

10. Clark CG (1997) Extensive genetic diversity in Blastocystis hominis. Mol Biochem Parasitol 87: 79-83.

11. Hoevers J, Holman P, Logan K, Hommel M, Ashford R, et al. (2000) Restriction-fragment-length polymorphism analysis of small-subunit rRNA genes of Blastocystis hominis isolates from geographically diverse human hosts. Parasitol Res 86 :57-61.

12. Böhm-Gloning B, Knobloch J, Walderich B (1997) Five subgroups of Blastocystis hominis from symptomatic and asymptomatic patients revealed by restriction site analysis of PCR-amplified 16S-like rDNA. Trop Med Int Health 2: 771-778.

13. Kaneda Y, Horiki N, Cheng XJ, Fujita Y, Maruyama M, et al. (2001) Ribodemes of Blastocystis hominis isolated in Japan. Am J Trop Med Hyg 65: 393-396.

14. Rivera WL, Tan MA (2005) Molecular characterization of Blastocystis isolates in the Philippines by riboprinting. Parasitol Res 96: 253-257.

15. Stensvold CR, Traub RJ, von Samson-Himmelstjerna G, Jespersgaard C, Nielsen HV, et al. (2007) Blastocystis: subtyping isolates using pyrosequencing technology. Exp Parasitol 116: 111-119.

16. Abe N, Wu Z, Yoshikawa H (2003) Molecular characterization of Blastocystis isolates from birds by PCR with diagnostic primers and restriction fragment length polymorphism analysis of the small subunit ribosomal RNA gene. Parasitol Res 89: 393-396.

17. Yan Y, Su S, Lai R, Liao H, Ye J, et al. (2006) Genetic variability of Blastocystis hominis isolates in China. Parasitol Res 99: 597-601.

18. Yoshikawa H, Wu Z, Kimata I, Iseki M, Ali IK, et al. (2004) Polymerase chain reaction-based genotype classification among human Blastocystis hominis populations isolated from different countries. Parasitol Res 92: 22-29.

19. Ronaghi M (2001) Pyrosequencing sheds light on DNA sequencing. Genome Res 11: 3-11.

20. Ahmadian A, Ehn M, Hober S (2006) Pyrosequencing: history, biochemistry and future. Clin Chim Acta 363: 83-94.

21. Jones MS 2nd, Ganac RD, Hiser G, Hudson NR, Le A, et al. (2008) Detection of Blastocystis from stool samples using real-time PCR Parasitol Res 103: 551-557.

22. Poirier P, Wawrzyniak I, Albert A, El Alaoui H, Delbac F, et al. (2011) Development and evaluation of a real-time PCR assay for detection and quantification of blastocystis parasites in human stool samples: prospective study of patients with hematological malignancies. J Clin Microbiol 49: 975-983.

23. Stensvold CR, Ahmed UN, Andersen LO, Nielsen HV (2012) Development and evaluation of a genus-specific, probe-based, internalprocess-controlled real-time PCR assay for sensitive and specific detection of Blastocystis spp. J Clin Microbiol 50: 1847-1851.

24. Stensvold CR, Alfellani M, Clark CG (2012) Levels of genetic diversity vary dramatically between Blastocystis subtypes. Infect Genet Evol 12: 263-273.

25. Hebert PD, Penton EH, Burns JM, Janzen DH, Hallwachs W (2004) Ten species in one: DNA barcoding reveals cryptic species in the neotropical skipper butterfly Astraptes fulgerator. Proceedings of the National Academy of Sciences of the United States of America101:14812-14817.

26. Hebert PD, Stoeckle MY, Zemlak TS, Francis CM (2004) Identification of Birds through DNA Barcodes. PLoS Biol 2: e312.

27. Stensvold CR (2013) Comparison of sequencing (barcode region) and sequence-tagged-site PCR for Blastocystis subtyping. J Clin Microbiol 51: 190-194.

28. Martiny D, Bart A, Vandenberg O, Verhaar N, Wentink-Bonnema E, et al. (2013) Subtype determination of Blastocystis isolates by matrixassisted laser desorption/ionisation time-of-flight mass spectrometry (MALDI-TOF MS). Eur J Clin Microbiol Infect Dis.

29. Stenzel DJ, Boreham PF (1996) Blastocystis hominis revisited. Clin Microbiol Rev 9: 563-584.

30. Stensvold CR, Arendrup MC, Jespersgaard C, Mølbak K, Nielsen HV (2007) Detecting Blastocystis using parasitologic and DNA-based methods: a comparative study. Diagn Microbiol Infect Dis 59: 303-307.

31. Roberts T, Barratt J, Harkness J, Ellis J, Stark D (2011) Comparison of microscopy, culture, and conventional polymerase chain reaction for detection of blastocystis sp. in clinical stool samples. Am J Trop Med Hyg 84: 308-312.

32. Souppart L, Sanciu G, Cian A, Wawrzyniak I, Delbac F, et al. (2009) Molecular epidemiology of human Blastocystis isolates in France. Parasitol Res 105: 413-421. 
33. Thathaisong U, Worapong J, Mungthin M, Tan-Ariya P, Viputtigul K, et al. (2003) Blastocystis isolates from a pig and a horse are closely related to Blastocystis hominis. J Clin Microbiol 41: 967-975.

34. Malheiros AF, Stensvold CR, Clark CG, Braga GB, Shaw JJ (2011) Short report: Molecular characterization of Blastocystis obtained from members of the indigenous Tapirapé ethnic group from the Brazilian Amazon region, Brazil. Am J Trop Med Hyg 85: 1050-1053.

35. Domínguez-Márquez MV, Guna R, Muñoz C, Gómez-Muñoz MT, Borrás R (2009) High prevalence of subtype 4 among isolates of Blastocystis hominis from symptomatic patients of a health district of Valencia (Spain). Parasitol Res 105: 949-955.

36. Lee IL, Tan TC, Tan PC, Nanthiney DR, Biraj MK, et al. (2012) Predominance of Blastocystis sp. subtype 4 in rural communities, Nepal. Parasitol Res 110: 1553-1562.

37. Ramírez JD, Sánchez LV2, Bautista DC2, Corredor AF2, Flórez AC3, et al. (2014) Blastocystis subtypes detected in humans and animals from Colombia. Infect Genet Evol 22: 223-228.

38. Wong KH, Ng GC, Lin RT, Yoshikawa H, Taylor MB, et al. (2008) Predominance of subtype 3 among Blastocystis isolates from a major hospital in Singapore. Parasitol Res 102: 663-670.

39. Li LH, Zhou XN, Du ZW, Wang XZ, Wang LB, et al. (2007) Molecular epidemiology of human Blastocystis in a village in Yunnan province, China. Parasitol Int 56: 281-286.

40. El Safadi D, Meloni D, Poirier P, Osman M, Cian A, et al. (2013) Molecular epidemiology of Blastocystis in Lebanon and correlation between subtype 1 and gastrointestinal symptoms. Am J Trop Med Hyg 88: 1203-1206.

41. Abu-Madi MA, Behnke JM, Ismail A (2008) Patterns of infection with intestinal parasites in Qatar among food handlers and housemaids from different geographical regions of origin. Acta Trop 106: 213-220.

42. Noor Azian MY, San YM, Gan CC, Yusri MY, Nurulsyamzawaty Y, et al. (2007) Prevalence of intestinal protozoa in an aborigine community in Pahang, Malaysia. Trop Biomed 24: 55-62.

43. Graczyk TK, Shiff CK, Tamang L, Munsaka F, Beitin AM, et al. (2005) The association of Blastocystis hominis and Endolimax nana with diarrheal stools in Zambian school-age children. Parasitol Res 98: 38-43.

44. Sohail MR, Fischer PR (2005) Blastocystis hominis and travelers. Travel Med Infect Dis 3: 33-38.

45. Herwaldt BL, de Arroyave KR, Wahlquist SP, de Merida AM, Lopez AS, et al. (2001) Multiyear prospective study of intestinal parasitism in a cohort of Peace Corps volunteers in Guatemala. J Clin Microbiol 39: 34-42.

46. Pipatsatitpong D, Rangsin R, Leelayoova S, Naaglor T, Mungthin M (2012) Incidence and risk factors of Blastocystis infection in an orphanage in Bangkok, Thailand. Parasit Vectors 5: 37.

47. Roberts T, Stark D, Harkness J, Ellis J (2013) Subtype distribution of Blastocystis isolates identified in a Sydney population and pathogenic potential of Blastocystis. Eur J Clin Microbiol Infect Dis 32: 335-343.

48. Nagel R, Cuttell L, Stensvold CR, Mills PC, Bielefeldt-Ohmann H, et al. (2012) Blastocystis subtypes in symptomatic and asymptomatic family members and pets and response to therapy. Intern Med J 42: 1187-1195.

49. Stensvold R, Brillowska-Dabrowska A, Nielsen HV, Arendrup MC (2006) Detection of Blastocystis hominis in unpreserved stool specimens by using polymerase chain reaction. J Parasitol 92: 1081-1087.
50. Stensvold CR, Lewis HC, Hammerum AM, Porsbo LJ, Nielsen SS, et al. (2009) Blastocystis: unravelling potential risk factors and clinical significance of a common but neglected parasite. Epidemiol Infect 137: 1655-1663.

51. Stensvold CR, Christiansen DB, Olsen KE, Nielsen HV (2011) Blastocystis sp. subtype 4 is common in Danish Blastocystis-positive patients presenting with acute diarrhea. Am J Trop Med Hyg 84: 883-885.

52. Hussein EM, Hussein AM, Eida MM, Atwa MM (2008) Pathophysiological variability of different genotypes of human Blastocystis hominis Egyptian isolates in experimentally infected rats. Parasitol Res 102: 853-860.

53. Fouad SA, Basyoni MM, Fahmy RA, Kobaisi MH (2011) The pathogenic role of different Blastocystis hominis genotypes isolated from patients with irritable bowel syndrome. Arab J Gastroenterol 12: 194-200.

54. Menounos PG, Spanakos G, Tegos N, Vassalos CM, Papadopoulou C, et al. (2008) Direct detection of Blastocystis sp. in human faecal samples and subtype assignment using single strand conformational polymorphism and sequencing. Molecular and Cellular Probes 22 :24-29.

55. Meloni D, Sanciu G, Poirier P, El Alaoui H, Chabé M, et al. (2011) Molecular subtyping of Blastocystis sp. isolates from symptomatic patients in Italy. Parasitol Res 109: 613-619.

56. Motazedian H, Ghasemi H, Sadjjadi SM (2008) Genomic diversity of Blastocystis hominis from patients in southern Iran. Ann Trop Med Parasitol 102: 85-88.

57. Moosavi A, Haghighi A, Mojarad EN, Zayeri F, Alebouyeh M, et al. (2012) Genetic variability of Blastocystis sp. isolated from symptomatic and asymptomatic individuals in Iran. Parasitol Res 111: 2311-2315.

58. Tan TC, Ong SC, Suresh KG (2009) Genetic variability of Blastocystis sp. isolates obtained from cancer and HIV/AIDS patients. Parasitol Res 105: 1283-1286.

59. Yoshikawa H, Wu Z, Pandey K, Pandey BD, Sherchand JB, et al. (2009) Molecular characterization of Blastocystis isolates from children and rhesus monkeys in Kathmandu, Nepal. Vet Parasitol 160: 295-300.

60. Bart A, Wentink-Bonnema EM, Gilis H, Verhaar N, Wassenaar CJ, et al. (2013) Diagnosis and subtype analysis of Blastocystis sp. in 442 patients in a hospital setting in the Netherlands. BMC Infect Dis 13: 389.

61. Rivera WL (2008) Phylogenetic analysis of Blastocystis isolates from animal and human hosts in the Philippines. Vet Parasitol 156: 178-182.

62. Forsell J, Granlund M, Stensvold CR, Clark CG, Evengård B (2012) Subtype analysis of Blastocystis isolates in Swedish patients. Eur J Clin Microbiol Infect Dis 31: 1689-1696.

63. Leelayoova S, Siripattanapipong S, Thathaisong U, Naaglor T, Taamasri P, et al. (2008) Drinking water: a possible source of Blastocystis spp. subtype 1 infection in schoolchildren of a rural community in central Thailand. Am J Trop Med Hyg 79: 401-406.

64. Dogruman-AF, Dagci H, Yoshikawa H, Kurt O, Demirel M (2008) A possible link between subtype 2 and asymptomatic infections of Blastocystis hominis. Parasitol Res 103: 685-689.

65. Ozyurt M, Kurt O, Mølbak K, Nielsen HV, Haznedaroglu T, et al. (2008) Molecular epidemiology of Blastocystis infections in Turkey. Parasitol Int 57: 300-306.

66. Eroglu F, Koltas IS (2010) Evaluation of the transmission mode of B. hominis by using PCR method. Parasitol Res 107: 841-845. 